\title{
Characteristics of a Magnetic Refrigerator with New Magnetocaloric Materials
}

\author{
Yoshiki MIYAZAKI Koichiro WAKI Yuki ARAI \\ Cryogenic Systems Laboratory, Maglev Systems Technology Division
}

Katsutoshi MIZUNO

Keisuke YOSHIZAWA

Cryogenic Systems Laboratory, Maglev Systems Technology Division

Ken NAGASHIMA

Maglev Systems Technology Division

\begin{abstract}
The aim of this study is to put a cooling technology using a magnetocaloric effect to practical use for air conditioners of railway vehicles in order to save energy. New magnetcaloric materials which have larger a magnetocaloric effect compared with Gd have been developed in the region of room temperature. Nevertheless, little discussion exists about how much the new materials affect the properties of a magnetocaloric cooling system. Therefore, the characteristics of the cooling system with $\mathrm{Gd}$ and $\mathrm{La}\left(\mathrm{Fe}_{0.84} \mathrm{Co}_{0.06} \mathrm{Si}_{0.10}\right)_{13}$ have been studied experimentally and analytically.
\end{abstract}

Keywords: air conditioner, magnetic refrigerator, Gd, LaFeCoSi, cooling capacity, COP

\section{Introduction}

Present air conditioner systems use traditional vapor compression technology with HCFC (Hydro Chloro Fluoro Carbon). The Montreal Protocol has obliged us to carry out the staged abolition of the CFC production in order to protect the Ozone layer. The Kyoto Protocol has designated HCFC as one of the gases whose emissions are to be reduced. This requires the development of HCFC free systems or usage of substances which have little greenhouse effect. On the other hand, the demands for reduction of environmental impact and energy conservation have become more stringent. Therefore, research and development is being conducted to produce air-conditioning systems with less influence on the environment [1].

Magnetic refrigeration technology which has the potential for high efficiency without Freon gases is a focus of attention.

The aim of this study is to development a magnetic refrigerator which has a maximum kilowatt-class cooling power and a COP (Coefficient of Performance) of over 6, utilizing the magnetocaloric effect, from the viewpoint of energy conservation of air-conditioners mounted on railway vehicles.

Magnetic refrigeration is a method utilizing the magnetocaloric effect, which is an entropy change when a magnetic material is magnetized or demagnetized. The compression and expansion processes of the gas cycle correspond to the magnetization and demagnetization processes of the magnetic refrigeration cycle, respectively. Magnetic materials play the role of the working gas of the gas cycle.

Since the heat capacity of magnetocaloric materials is large at room temperature, temperature change available for refrigeration is small. Therefore, for practical use, a stronger magnetic field is necessary to enlarge the temperature change due to the magnetocaloric effect. The working range of magnetocaloric materials is limited around its $\mathrm{Cu}$ rie point. Therefore, it is proposed that several materials which have different Curie points be used in order to obtain a wider temperature range. However it is not known sufficiently how the characteristics of magnetocaloric materials affect the magnetic refrigerator system.

In this paper, the results of the cooling capacity and power consumption of the magnetic refrigerator system using gadolinium $(\mathrm{Gd})$ and $\mathrm{La}\left(\mathrm{Fe}_{0.84} \mathrm{Co}_{0.06} \mathrm{Si}_{0.10}\right)_{13}$ (from here on abbreviated as "LaFeCoSi" ) are discussed. Gd is generally used as a magnetic material at room temperature range, while LaFeCoSi is a new material which has a large entropy change compared with Gd.

\section{Magnetic material and refrigeration cycle}

\subsection{Entropy change of magnetic materials}

Gd and LaFeCoSi are second-order phase-transition materials which have Curie points of $293 \mathrm{~K}$ and $281 \mathrm{~K}$ respectively. The important properties are magnetic entropy change, specific heat and adiabatic temperature change. These properties are compared as follows. The properties

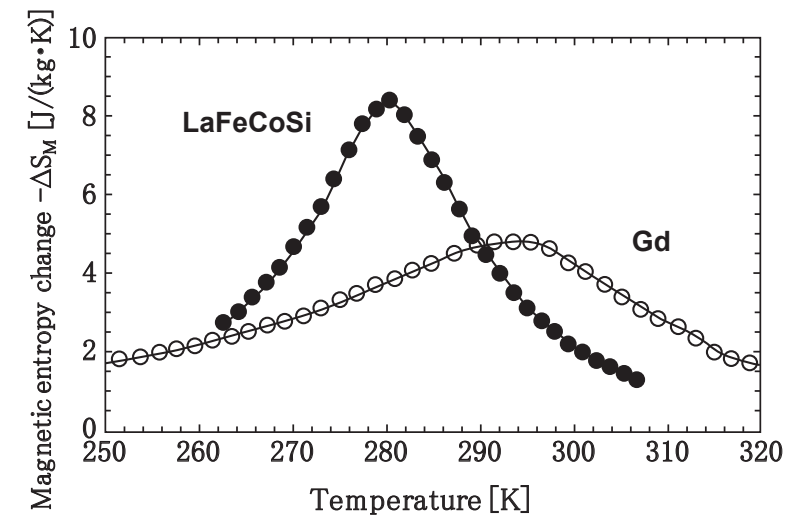

Fig. 1 Magnetic entropy change $\Delta S M$ for Gd and LaFe$\mathrm{CoSi}$ in a magnetic field change of 0-2 T. 
of Gd mentioned in Reference [2] were used. The material properties of LaFeCoSi are compared with that of Gd. Magnetic entropy change is estimated by measuring magnetization as a function of the temperature.

Figure 1 shows the temperature dependence of magnetic entropy change (0-2 T) of Gd and LaFeCoSi. Gd has a maximum magnetic entropy change of $5 \mathrm{~J} /(\mathrm{kg} \mathrm{K})$ at around $293 \mathrm{~K}$, which is the Curie point of Gd. LaFeCoSi has a maximum magnetic entropy change of $8 \mathrm{~J} /(\mathrm{kg} \mathrm{K})$ at around $281 \mathrm{~K}$, which is the Curie point of this material.

\subsection{Specific heat and adiabatic temperature change in magnetic materials}

Figure 2 shows specific heat of Gd and LaFeCoSi. LaFeCoSi has large specific heat compared with that of Gd. When the field changes adiabatically, the temperature change of the magnetic material is called the adiabatic temperature change.

By measuring specific heat, the temperature change can be derived from the entropy change using the following formula:

$$
\Delta T_{a d}=-\frac{\Delta S_{M} T}{c}
$$

$\Delta T_{a d}$ : Adiabatic temperature change, $\Delta S_{M}$ : Magnetic entropy change, $T$ : Temperature, $c$ : specific heat

Figure 3 shows the adiabatic temperature change of Gd and LaFeCoSi. The temperature change of LaFeCoSi is smaller than that of Gd due to the large specific heat of $\mathrm{LaFeCoSi}$ compared with that of Gd.

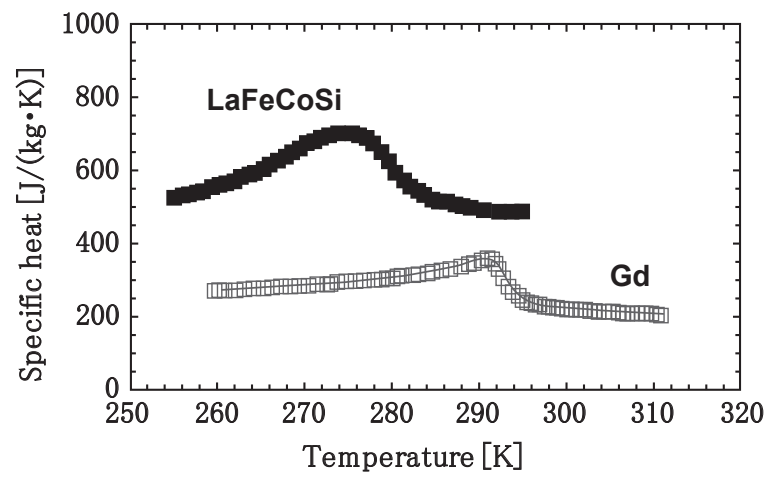

Fig. 2 Specific heat for Gd and LaFeCoSi in a magnetic field change of 0-2 T.

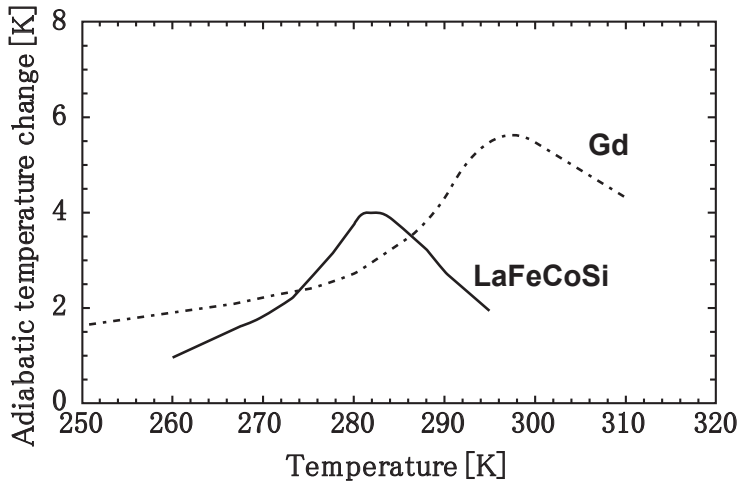

Fig. 3 Adiabatic temperature change for Gd and LaFeCoSi in a magnetic field change of 0-2 T.

\subsection{Refrigeration Cycle}

Barclay (1981) proposed the AMR (Active Magnetic Regenerator) cycle in order to obtain a large temperature change with a small magnetic field of a permanent magnet [3]. The AMR cycle can obtain a large temperature span by "storing" the temperature change of the material, using the material as a regenerator. The AMR is thought to be the most effective method for magnetic refrigeration at room temperature. In this paper, the AMR cycle is adopted for the refrigeration cycle.

The principle of the AMR cycle is shown in Fig. 4. The temperature of magnetic materials rises with adiabatic magnetization in the first process (a). In the next process (b), the temperature incline occurs in the material because of the heat exchange with cold fluid (e.g. water). Subsequently, the temperature of the material drops while maintaining its temperature incline in the third process (c). Finally, the temperature incline is increased by exchanging heat with hot fluid in the fourth process $(d)$. The casing of the magnetic material is called the AMR bed.

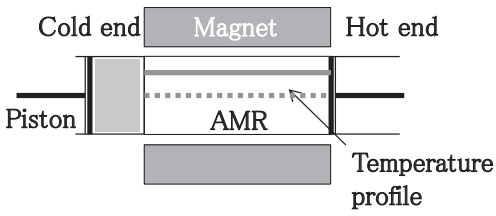

(a) Adiabatic magnetization process

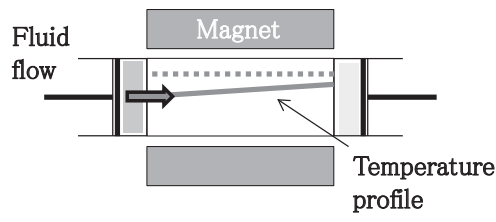

(b) Heat exchange process

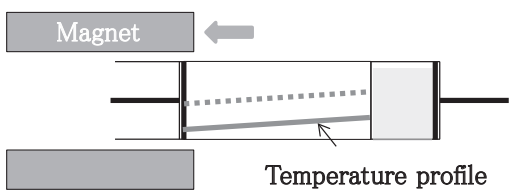

(c) Adiabatic demagnetization process

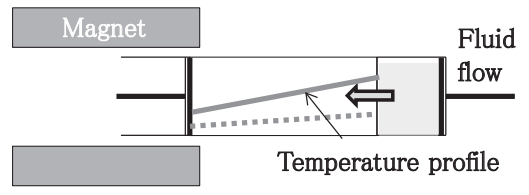

(d) Heat exchange process

Fig. 4 Schematic of the Active Magnetic Regenerator.

\subsection{Magnetic refrigerator}

The prototype of the magnetic refrigerator has a magnetic circuit which consists of 12 fixed AMR beds (Fig. 5) and a ring-shaped Halbach-arrayed permanent-magnet assembly rotating over the AMR beds [4]. Each bed was packed with Gd or LaFeCoSi particles.

Figure 6 shows the schematic of the magnetic refrigerator. The Halbach-arrayed permanent magnet is shaped 


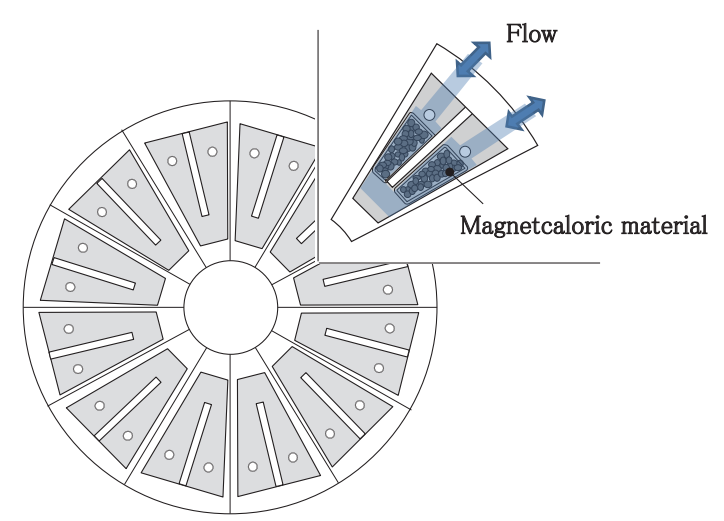

Fig. 5 The AMR beds.

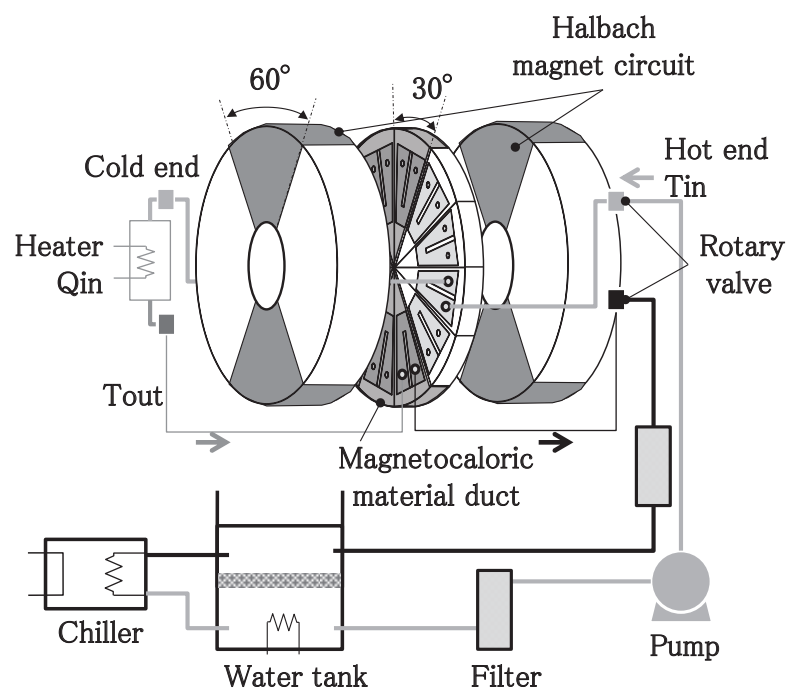

Fig. 6 Schematic of the magnetic regeneration system with the AMR beds.

like a ring, which consists of two main magnets and two sub-magnets. The main magnets hold the axial magnetic poles. The Halbach-arrayed magnet can magnetize and demagnetize the magnetic materials in the AMR beds effectively because the circumferentially-magnetized submagnets can augment the magnetic field of the poles of the main magnets.

(1)The fluid for heat exchange at the temperature of $T_{\text {in }}$ is transferred from the pump to the AMR beds. After exchanging heat, the fluid temperature drops.

(2)The fluid absorbs the heat load " $Q_{i n}$ " of a heater,

\begin{tabular}{cc} 
Table 1 Specifications of the magnetic refrigerato \\
\hline Magnetic material & $\mathrm{Gd}, \mathrm{LaFeCoSi}$ \\
$\varphi 0.6 \mathrm{~mm}$ \\
Packing mass & $0.75 \mathrm{~kg}$ \\
Number of AMR bed & 12 \\
Size & $20 \mathrm{~mm} \times 10 \mathrm{~mm} \times 76 \mathrm{~mm}$ \\
Flow rate & $\sim 5 \mathrm{liter} / \mathrm{min}^{-1}$ \\
Rotation number & $\sim 60 \mathrm{~min}$ \\
Permanent magnet & $\mathrm{NeFeB}$ \\
Bmax & $1.1 \mathrm{~T}$ \\
\hline
\end{tabular}

and the temperature becomes $T_{\text {out }}$. Then the fluid is transferred to the AMR beds in the process of magnetization.

(3)After the fluid absorbs the heat of the magnetic material, the temperature is reset to $T_{i n}$ by a chiller.

To carry out the above cycle in each AMR bed, the flow channels of the fluid are changed by the rotary valves which are synchronized with the rotation of the magnet. The specifications of the magnetic refrigerator are shown in Table 1.

\section{Results of experiments}

\subsection{Comparing the cooling capacity}

$T_{\text {in }}$ was set to the Curie point of each material, at $20^{\circ} \mathrm{C}$ and $8^{\circ} \mathrm{C}$ in the cases of $\mathrm{Gd}$ and $\mathrm{LaFeCoSi}$, respectively. The cooling capacity is defined as the heat load of the heater. The temperature span is defined as $T_{i n}-T_{\text {out }}$. The cooling capacity was measured in the condition of $0 \mathrm{~K}$ temperature span, in order to assess the differing characteristics of the system due to the differences in materials of $\mathrm{Gd}$ and LaFeCoSi. Figures 7 and 8 show the cooling capacity as a function of the volumetric flow rate of $\mathrm{Gd}$ and $\mathrm{LaFeCoSi}$, respectively.

The cooling capacity increased with the increase of volumetric flow rate. When the number of rotations is $5 \mathrm{~min}^{-1}$, the cooling capacity of Gd has a peak at a volumetric flow rate of $2 \mathrm{~L} / \mathrm{min}$. The heat exchange between the fluid and magnetic material increases as fluid flow through the AMR beds rises. Therefore, the cooling capacity increases up to a certain flow rate. However, since the temperature change of the fluid becomes small if the volumetric flow rate is increased, the cooling capacity decreases when the flow rate increases beyond the point of sufficient heat exchange.

In the cases of the number of rotations of 5, 10, $40 \mathrm{~min}^{-1}$ for $\mathrm{LaFeCoSi}$, the cooling capacity increased in the range of 0-5 L/min. Compared with the results for Gd, the cooling capacity of LaFeCoSi is larger than that of Gd when the number of rotations is of 5 and $10 \mathrm{~min}^{-1}$; however, the cooling capacity is smaller than that of Gd when the number of

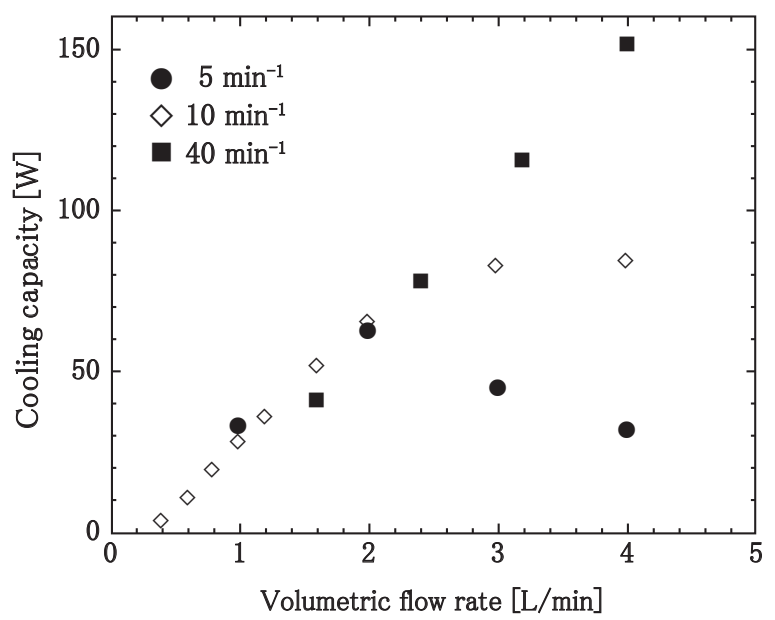

Fig. 7 Cooling capacity of the magnetocaloric refrigerator system for Gd, as a function of volumetric flow rate. 


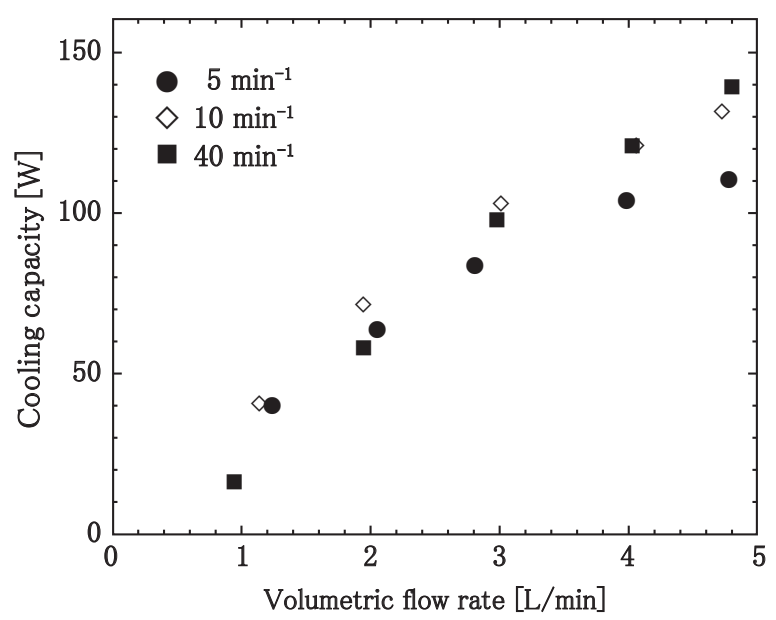

Fig. 8 Cooling capacity of the magnetocaloric refrigerator system for LaFeCoSi, as a function of volumetric flow rate.

rotations is $40 \mathrm{~min}^{-1}$. The rate at which the cooling capacity of $\mathrm{LaFeCoSi}$ increases with the rise in volumetric flow rate is smaller than that of Gd when the number of rotations is increased.

The results of the differing cooling capacities of $\mathrm{Gd}$ and LaFeCoSi can be understood by taking into consideration the difference in the specific heat. Because the specific heat of $\mathrm{LaFeCoSi}$ is larger than that of $\mathrm{Gd}$, not all of the heat of the magnetic material is thought to be absorbed by the heat transfer fluid in the range of $0-5 \mathrm{~L} / \mathrm{min}$. The heat exchanging time becomes short by increasing the number of rotations because the rotations of the rotary valves are synchronized with the rotation of the magnet. In the case of LaFeCoSi as the magnetic material, the shorter the heat exchanging time in the region of low volumetric flow rate, the smaller the heat exchange. Therefore, even if the number of rotations is increased, the effect on the cooling capacity is small.

\subsection{Power of the motor and pump}

The power of both the motor and the pump of the system using Gd are compared with that using LaFeCoSi in Fig. 9. The power of the motor necessary for rotating the magnet is estimated by the number of rotations and the torque. The power of the pump necessary for circulating the heat transfer fluid is estimated by the volumetric flow rate and the pressure drop. The power of the motor, irrespective of whether Gd or LaFeCoSi is used, has a constant value because the torque does not depend on the volumetric flow rate. The power of the pump using $\mathrm{Gd}$ is about the same level as that using LaFeCoSi.

\section{Numerical analysis}

\subsection{Characteristics of the cooling capacity of the prototype}

The flow rate of the system is limited to the range of $0-5 \mathrm{~L} / \mathrm{min}$ because the pressure of flow rates beyond this

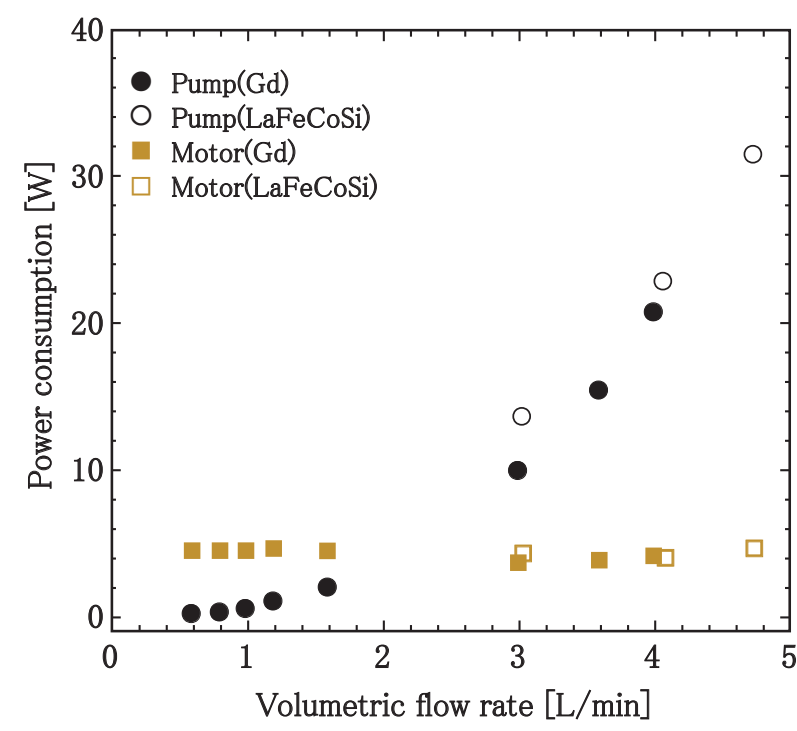

Fig. 9 Power consumption of the motor and pump

range would exceed the pressure resistance limit of the AMR bed; therefore, the behavior of the cooling capacity is unknown for higher flow rates. The cooling capacity of $\mathrm{Gd}$ and LaFeCoSi when the temperature span is zero is estimated by numerical analysis. The energy balance of the magnetic material and the heat transfer fluid in the AMR are expressed in Equations (2) and (3), respectively, as follows:

The magnetic material (suffix “ $s$ ");

$$
\rho_{s} c_{p s} \frac{\partial T_{s}}{\partial t}=\lambda_{s} A \frac{\partial T_{s}}{\partial x^{2}}+\frac{A}{V_{s}} h\left(T_{f}-T_{s}\right)+\frac{Q_{m a g}}{\Delta t V_{s}}
$$

The heat transfer fluid (suffix " $f$ ");

$$
\rho_{f} c_{p f}\left(\frac{\partial T_{f}}{\partial t}+u^{\prime} \frac{\partial T_{f}}{\partial x}\right)=\lambda_{s} A \frac{\partial^{2} T_{f}}{\partial x^{2}}+\frac{A}{V_{f}} h\left(T_{s}-T_{f}\right)
$$

$\rho$ : Density, $c$ : specific heat, $T$ : Temperature, $\lambda$ : Thermal conductivity, $\Delta t$ : Heat exchanging time, $h$ : Heat transfer coefficient, $x$ : Length of the flow channel, $A$ : Surface of the flow channel, $V$ : Volume of the flow channel, $Q_{\text {mag }}$ : magnetocaloric effect, $u^{\prime}$ : velocity

The cooling capacity as a function of the number of rotations and the flow rates was calculated by Equations (2) and (3).

Figure 10 shows the result of the calculation for Gd. In the experiments, the peak of the cooling capacity was observable only when the number of rotations was $5 \mathrm{~min}^{-1}$. In the numerical analysis, the peak of the cooling capacity was observed when the number of rotations was $10 \mathrm{~min}^{-1}$.

Figure 11 shows the result of the calculation for LaFeCoSi. The cooling capacity of LaFeCoSi has peak values when the number of rotations is 5 and $10 \mathrm{~min}^{-1}$. These peak values were larger than those of Gd. For the number of rotations of $40 \mathrm{~min}^{-1}$, the cooling capacity of LaFeCoSi is larger than that of Gd. Because of the large heat capacity, LaFeCoSi can maintain its temperature span.

In Fig. 12, the cooling capacity of $\mathrm{Gd}$ and LaFeCoSi are shown in contour maps, in order to compare the two magnetic materials in terms of the relationship between the volumetric flow rate and the number of rotations of 


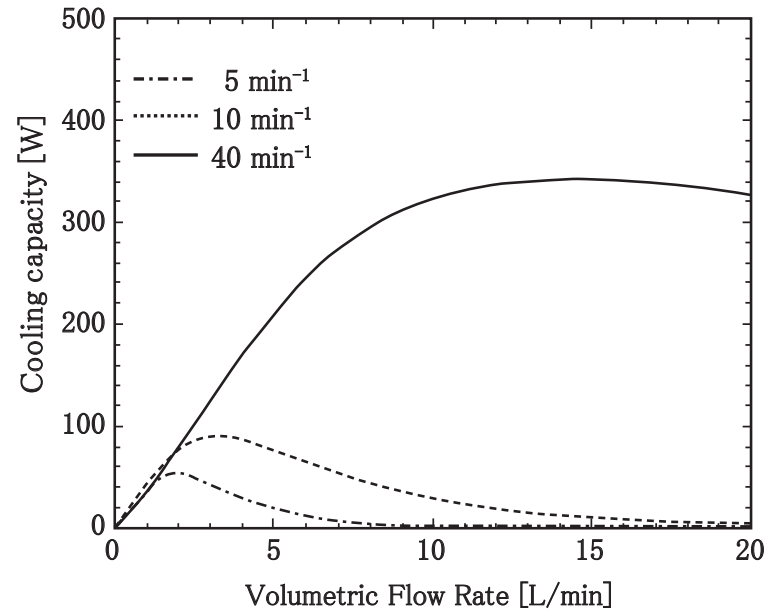

Fig. 10 Calculated cooling power of the magnetocaloric refrigerator system with $\mathrm{Gd}$ as a function of volumetric flow rate.

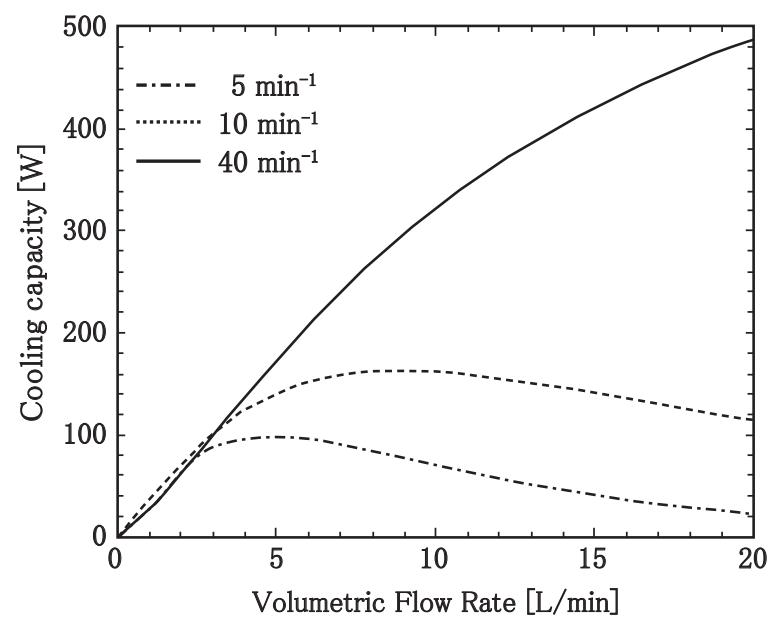

Fig. 11 Calculated cooling power of the magnetocaloric refrigerator system with $\mathrm{LaFeCoSi}$ as a function of volumetric flow rate.

the magnet. The values shown in this figure at the borders between regions of different colors represent the cooling capacity at those borders.

The cooling capacity is large in regions of high flow rates in the cases of both $\mathrm{Gd}$ and $\mathrm{LaFeCoSi}$. However, there is a difference between $\mathrm{Gd}$ and $\mathrm{LaFeCoSi}$ in terms of the region of the number of rotations where the cooling

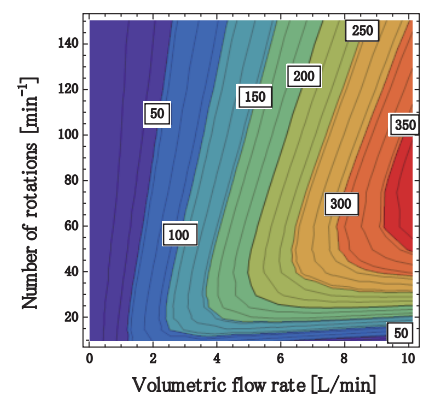

(a) Gd

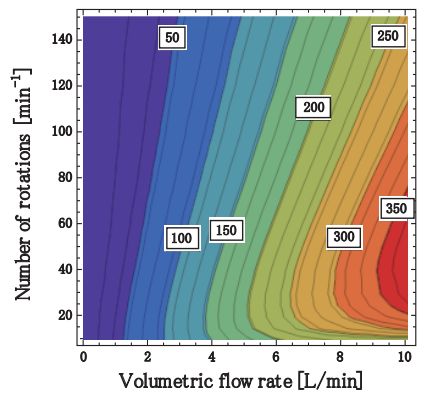

(b) $\mathrm{LaFeCoSi}$
Fig. 12 Calculated cooling capacity for Gd and LaFeCoSi capacity becomes large. The number of rotations is larger in the case of Gd than in the case of $\mathrm{LaFeCoSi}$. The specific heat of $\mathrm{Gd}$ is smaller than that of LaFeCoSi. Therefore, the heat exchanging time for Gd can be shorter than for LaFeCoSi. To obtain a large cooling capacity, a higher flow rate and a larger number of rotations are required. However, the appropriate flow rate and number of rotations should be selected in accordance with the materials.

\subsection{Amount of magnetic material packed into the AMR beds}

The larger the amount of magnetic material in the AMR bed, the larger the heat capacity. Therefore a large heat capacity flow of the heat transfer fluid is needed. Figure 13 shows the cooling capacity when Gd is packed in the AMR bed, in the cases of $0.75 \mathrm{~kg}$ and $0.375 \mathrm{~kg}$. Both results show that the cooling capacity tends to increase with the increase of the volumetric flow rate. However, the number of rotations of the motor that corresponds to the cooling capacity peak in the case of $0.375 \mathrm{~kg}$ of Gd is larger compared with in the case of $0.75 \mathrm{~kg}$ of Gd (with the number of rotations being proportional to the heat exchanging time).

Figure 14 shows the maximum cooling capacity per kilogram in the case of $0.75 \mathrm{~kg}$ and $0.375 \mathrm{~kg}$ of Gd. From a viewpoint of material efficiency, it is effective to increase the cooling capacity by means of the quick heat exchange of a small amount of material and the increase of the number of rotations of the magnet.

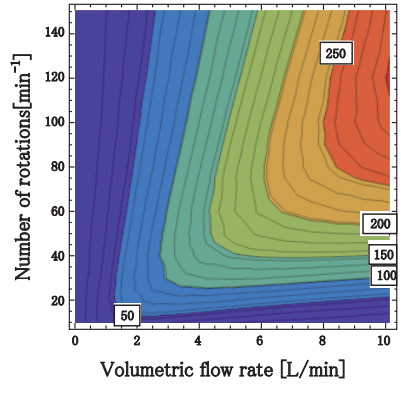

(a)packed $0.375 \mathrm{~kg}$

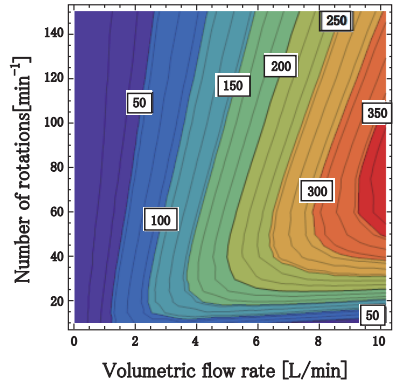

(b)packed $0.750 \mathrm{~kg}$
Fig. 13 Calculated cooling capacity for Gd

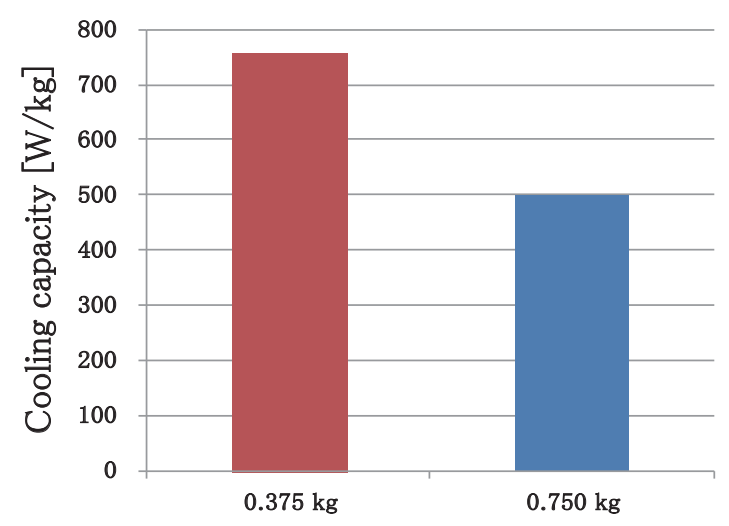

Fig. 14 Maximum cooling capacity of Gd (calculation) 


\section{Conclusions}

Gd and LaFeCoSi were the magnetic materials packed in the AMR beds. The cooling capacity and power consumption of these materials were evaluated. Furthermore, the experimental results were supplemented and confirmed by numerical analysis.

As far as the scope of the flow rates which the experiments covered is concerned, the cooling capacity of LaFe$\mathrm{CoSi}$ is larger than that of Gd when the number of rotations of the magnet is 5 or $10 \mathrm{~min}^{-1}$. However, the cooling capacity of Gd is larger than that of LaFeCoSi when the number of rotations of the magnet is $40 \mathrm{~min}^{-1}$. The results of numerical analysis show that the cooling capacity of $\mathrm{LaFeCoSi}$ is larger than that of $\mathrm{Gd}$ in the region of high flow rates when the number of rotations is $40 \mathrm{~min}^{-1}$.

The cooling capacity of LaFeCoSi is superior to that of Gd, because LaFeCoSi which has large specific heat can maintain its temperature span. The larger the adiabatic temperature change of the magnetic material, the faster the speed of the heat exchange between the material and the fluid. Therefore, the system frequency can be increased and the efficiency improved. Because LaFeCoSi has large specific heat, it is important to examine to what extent the efficiency of the heat exchange can be improved in the case of LaFeCoSi. The numerical analysis of this work helped gain valuable perspectives on a system design that takes into consideration the characteristics of the materials.

\section{Acknowledgment}

This work was supported by the New Energy and Industrial Technology Development Organization (NEDO).

\section{References}

[1] http://www.meti.go.jp/policy/chemical_management/ ozone/law_furon_outline.html

[2] S. Yu, Dan'kov, A. M. Tishin et al., "Magnetic phase transitions and the magnetothermal properties of gadolimum," PHYSICAL REVIEW B, Vol. 57, No. 6, pp. 3478-3490, 1998.

[3] Barclay, J. A., Steyert, W. A., “Active Magnetic Regenerator," U.S. Patent, 4,332,135, 1981.

[4] K. Waki, Y. Miyazaki, Y. Arai, K. Mizuno, K. Nagashima, T. Kawanami, "Performance of room-temperature magnetic refrigerator applying Ring Halbach-array Magnetic Circuit,” RTRI Report, Vol. 26, No. 5, pp. 47 52, 2012 (in Japanese).

\section{Authors}

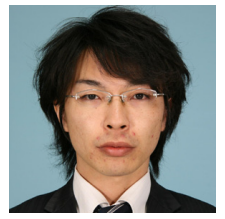

Yoshiki MIYAZAKI, Ph.D.

Assistant Senior Researcher, Cryogenic Systems Laboratory, Maglev Systems Technology Division

Research Areas: Cryogenics and Superconductivity

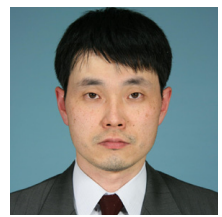

Koichiro WAKI

Senior Researcher, Cryogenic Systems Laboratory, Maglev Systems Technology Division

Research Areas: Cryogenics and Superconductivity

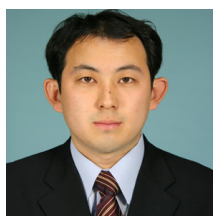

Yuuki ARAI

Assistant Senior Researcher, Cryogenic Systems Laboratory, Maglev Systems Technology Division

Research Areas: Cryogenics and

Superconductivity

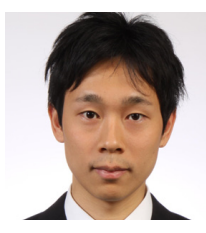

Katsutoshi MIZUNO

Researcher, Cryogenic Systems

Laboratory, Maglev Systems Technology Division

Research Areas: Cryogenics and Superconductivity

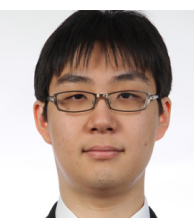

\section{Keisuke YOSHIZAWA}

Researcher, Cryogenic Systems Laboratory, Maglev Systems Technology Division

Research Areas: Cryogenics and Superconductivity

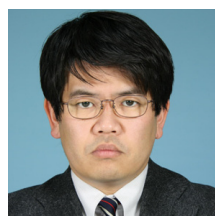

Ken NAGASHIMA, Ph.D.

Director, Maglev Systems Technology

Division

Research Areas: Cryogenics and

Superconductivity 